\title{
Examples of exotic free 2-complexes and stably free nonfree modules for quaternion groups
}

\author{
F RUDOLF BEYL \\ NANCY WALLER
}

\begin{abstract}
This is a continuation of our study [3] of a family of projective modules over $Q_{4 n}$, the generalized quaternion (binary dihedral) group of order $4 n$. Our approach is constructive. Whenever $n \geq 7$ is odd, this work provides examples of stably free nonfree modules of rank 1, which are then used to construct exotic algebraic 2complexes relevant to Wall's $\mathrm{D}(2)-$ problem. While there are examples of stably free nonfree modules for many infinite groups $G$, there are few actual examples for finite groups. This paper offers an infinite collection of finite groups with stably free nonfree modules $P$, given as ideals in the group ring. We present a method for constructing explicit stabilizing isomorphisms $\theta: \mathbb{Z} G \oplus \mathbb{Z} G \cong P \oplus \mathbb{Z} G$ described by $2 \times 2$ matrices. This makes the subject accessible to both theoretical and computational investigations, in particular, of Wall's D(2)-problem.
\end{abstract}

16D40, 19A13, 57M20; 55P15

\section{Introduction}

Our motivation for constructing stably free nonfree modules is their relevance to the homotopy classification of 2-complexes, in particular, Wall's D(2)-problem [22; 23]:

D(2)-problem Suppose $X$ is a finite three-dimensional connected $C W$-complex (with universal cover $\tilde{X})$ such that $H_{3}(\tilde{X}, \mathbb{Z})=0$ and $H^{3}(X, \mathcal{B})=0$ for all local coefficient systems $\mathcal{B}$ on $X$. Is $X$ homotopy equivalent to a finite 2 -complex?

This is equivalent to showing that every chain homotopy type of algebraic 2-complexes is geometrically realizable, ie, the homotopy class contains a chain complex of the universal cover of a presentation complex for $G$ [9]. Johnson [8, Theorem IV, p 220] showed that the chain homotopy classes of algebraic 2-complexes of minimal Euler characteristic over $Q_{4 n}$ correspond to the isomorphism classes of rank 1 stably free $\mathbb{Z} Q_{4 n}$-modules. It is unknown whether the algebraic 2-complexes corresponding to stably free nonfree modules are geometrically realizable. If one such algebraic 
2-complex were not geometrically realizable, it would provide a negative answer to Wall's D(2)-problem.

Major techniques for classifying the homotopy types of presentation complexes actually classify chain homotopy types of algebraic 2-complexes; see eg, Beyl-Latiolais-Waller [2], Browning [4], Johnson [8] and Sieradski-Dyer [16]. As long as Wall's D(2)problem remains unresolved, a complete homotopy classification of 2-complexes requires that an actual presentation complex be constructed for each chain homotopy type. It is inconceivable to do this without explicitly describing the second boundary map of the algebraic 2-complex, which in turn relies on specifying a free basis for some stabilization of a stably free nonfree module, ie, a specific stabilizing isomorphism. In [3] we constructed a specific example of an algebraic 2-complex over $Q_{28}$ for which the geometric realization question remains unanswered, and we proposed it as a test object for Wall's D(2)-problem. A key ingredient of the argument is replacing the second homotopy module of a geometric complex by some $\mathbb{Z} G$-module $P / N P$. Here $N$ is the sum of the group elements and $P$ is required to be stably free. When $P / N P$ is not singly generated, the resulting algebraic 2-complex is called exotic. This paper provides the set of algebraic tools that allow one to generalize the previous construction to $\mathbb{Z} Q_{4 n}$ for all odd $n \geq 7$. This gives an infinite list of such test objects. Since some of the reasoning given in [3] does not extend to the general case without an excursion into class field theory, we here present independent and more comprehensive arguments that certain modules are stably free and not free. We have taken care to keep proofs as elementary as possible.

Let $G$ be a group. All $\mathbb{Z} G$-modules considered in this paper are assumed to be finitely generated. A $\mathbb{Z} G$-module $P$ is stably free if there is an isomorphism

$$
P \oplus \mathbb{Z} G^{\ell} \cong \mathbb{Z} G^{k} \oplus \mathbb{Z} G^{\ell}
$$

for some $k, \ell \geq 0$. If $P \cong \mathbb{Z} G^{k}$ whenever this condition is met, all stably free modules are free and $\mathbb{Z} G$ is said to have the cancellation property. Berridge-Dunwoody [1], Lewin [12] and also Harlander-Jensen [6] give examples of stably free nonfree modules for certain infinite groups. For most finite groups $G$, no such examples exist. The reason is that for such a group Jacobinski's Cancellation Theorem, as presented by SwanEvans [19, Theorem 9.9], implies that $\mathbb{Z} G$ has the cancellation property whenever $\mathbb{Z} G$ satisfies the Eichler condition. This happens whenever $G$ has no quotient that is one of the binary polyhedral groups: $Q_{4 n}$ for $n \geq 2, T_{24}, O_{48}, I_{120}$ [19, p 178f]. The earliest example of noncancellation for an integral group ring of a finite group, specifically $Q_{32}$, is due to Swan [18]. Based on Swan's results, Johnson [10] shows the existence of exotic algebraic 2-complexes over $Q_{2^{n}}$ for $n \geq 5$. Vignéras [21] showed that $\mathbb{Z} Q_{4 n}$ does not have the cancellation property when $n$ is sufficiently 
large. Swan [20] gave a sharper bound and systematically studied the remaining cases: The only binary polyhedral groups $G$ such that $\mathbb{Z} G$ has the cancellation property are $Q_{8}, Q_{12}, Q_{16}, Q_{20}, T_{24}, O_{48}$, and $I_{120}$. The results of Swan and Vignéras imply the existence of stably free nonfree modules for all other $\mathbb{Z} Q_{4 n}$, but without explicit construction.

In Section 4 we give specific examples, for all odd $n \geq 7$, of stably free nonfree modules over $Q_{4 n}$. Each such module is described as a left ideal of $\mathbb{Z} Q_{4 n}$ with two explicitly given generators, and belongs to a family of rank $1 \mathbb{Z} Q_{4 n}$-projectives $P$ for $n \geq 2$. By Theorem 1.6 the class of $P$ has order at most 2 in the reduced class group $C\left(\mathbb{Z} Q_{4 n}\right)=\widetilde{K}_{0}\left(\mathbb{Z} Q_{4 n}\right)$. Section 2 gives a simple condition for $P$ to be stably free and exhibits an explicit stabilizing isomorphism $\mathbb{Z} Q_{4 n} \oplus \mathbb{Z} Q_{4 n} \cong P \oplus \mathbb{Z} Q_{4 n}$ when this condition is met. Note that this provides a free basis for a stabilization of $P$. For odd $n$, Section 3 gives easily testable criteria for $P$ to be nonfree. Our results depend on a lemma of Magurn-Oliver-Vaserstein [13], which shows that the units in certain factor rings of $\mathbb{Z} Q_{4 n}$ have a relatively simple structure. For the purpose of constructing examples of exotic algebraic 2-complexes in Section 4, we also give conditions for $P / N P$ not to be singly generated as a $\mathbb{Z} Q_{4 n}$-module.

The reader may use the methods presented here to construct additional explicit examples of exotic algebraic 2-complexes. We hope that the results of this paper will lead to further investigations of the $\mathrm{D}(2)-$ problem of C T C Wall.

We gladly acknowledge inspiration from the work of R G Swan. We thank Cameron Gordon, Jens Harlander, Cynthia Hog-Angeloni, Wolfgang Metzler, and Kalathoor Varadarajan for their encouragement to publish these results in connection with Wall's D(2)-problem, and Konstantin Psouroukis for providing an additional reference.

\section{A class of projective modules over $\mathbb{Z} Q_{4 n}$}

Here the underlying group is the generalized quaternion (binary dihedral) group

$$
Q_{4 n}=\left\langle x, y \mid x^{n}=y^{2}=(x y)^{2}\right\rangle, \quad n \geq 2 .
$$

Given integers $a$ and $b$, not both zero, let $P=P_{a+b y}$ be the left ideal in $\mathbb{Z} Q_{4 n}$ generated by $a+b y$ and $x+1$. Let $k=a^{2}+b^{2}$ for $n$ odd, and $k=a^{2}-b^{2}$ for $n$ even. As we showed in [3, Proposition 2.1], this $P$ is actually a two-sided ideal and is projective whenever $(k, 2 n)=1$. From now on, assume that $(k, 2 n)=1$. Then both $k$ and $N$ are elements of $P$, where $N$ is the sum of the group elements.

Let $\Sigma^{-}=1-x+x^{2}-x^{3}+\cdots-x^{2 n-1}$ and let $\mathbb{Z}_{m}=\mathbb{Z} / m \mathbb{Z}$. Since $\left\langle\Sigma^{-}\right\rangle$and $\langle x+1\rangle$ are two-sided ideals with trivial intersection, the group ring $\mathbb{Z} Q_{4 n}$ may be 
decomposed by a bicartesian square of rings:

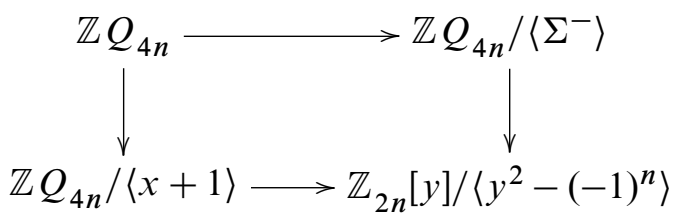

Overview We will be interested in avoiding elements of $\mathbb{Z} Q_{4 n}$ which lie over $a+b y \in$ $\mathbb{Z} Q_{4 n} /\langle x+1\rangle$ and are units in $\mathbb{Z} Q_{4 n} /\left\langle\Sigma^{-}\right\rangle$; cf Lemma 3.2. The scarcity of such elements implies that many $P_{a+b y}$ are not free; cf Theorem 3.11. On the other hand, there are sufficiently many units in $\mathbb{Z} Q_{4 n} /\left\langle\Sigma^{-}\right\rangle$to allow the construction of $2 \times 2$ matrices that are invertible mod $\left\langle\Sigma^{-}\right\rangle$. Hence many $P_{a+b y}$ are stably free; cf Theorem 2.2.

For the main purpose of this paper, all that is required is the existence of left inverses for certain matrices, but for completeness note the following.

Remark 1.1 Let $M_{\ell}(R)$ denote the ring of $\ell \times \ell$ matrices over the unital ring $R$. If $U \cdot V=1$ in $M_{\ell}(\mathbb{Z} G)$ for a group $G$, then also $V \cdot U=1$. In other words, if $V$ has a left inverse, it is invertible. (Rings with this property are called von Neumann finite.) This statement is highly nontrivial for infinite groups $G$ and in this case is known as Kaplansky's Theorem [11, p 122]; see also Bogley [7, p 328], Montgomery [14] and Passman [15, p 38]. Recall that for finite groups $G$ this follows from standard linear algebra, since the $\ell \times \ell$ matrices with entries in $\mathbb{Z} G$ can be interpreted as $\mathbb{Q}$-linear endomorphisms of the finite-dimensional rational vector space $M_{\ell}(\mathbb{Q} G)$. When $I$ is an ideal in $\mathbb{Z} G$, the same holds for $R=\mathbb{Z} G / I$ in place of $\mathbb{Z} G$, provided that $G$ is finite and $\mathbb{Z} G / I$ is torsion-free as an abelian group.

In this context, the following lemma will be used several times.

Lemma 1.2 Let $\Xi=(1+y) \sum_{\ell=0}^{n-1} x^{2 \ell}$ in $\mathbb{Z} Q_{4 n}$. Whenever $I$ is the ideal $\left\langle\Sigma^{-}\right\rangle$or $\langle\Xi\rangle$, then the additive group of $\mathbb{Z} Q_{4 n} / I$ is torsion-free.

Proof The ideal $\langle\Xi\rangle$ is actually two-sided because $\Xi$ is central in $\mathbb{Z} Q_{4 n}$. In each case, there is a basis for $\mathbb{Z} Q_{4 n}$ as a free abelian group which contains a basis for $I$, hence the complement of the basis for $I$ gives a free basis for $\mathbb{Z} Q_{4 n} / I$. In particular, $\left\langle\Sigma^{-}\right\rangle$has rational rank 2 . And $\langle\Xi\rangle$ has rational rank 3 or 2 , depending on whether $n$ is odd or even, respectively.

Algebraic 83 Geometric Topology, Volume 8 (2008) 
Lemma 1.3 For $m \geq 1$, let

$$
u_{m}=\sum_{i=0}^{m-1}(-x)^{i}
$$

If $(m, 2 n)=1$, then $u_{m}$ is a unit $\bmod \left\langle\Sigma^{-}\right\rangle$and $z_{m}=(-x)^{-\frac{m-1}{2}} u_{m}$ is central in $\mathbb{Z} Q_{4 n}$.

Proof Let $\ell \geq 1$ be an integer such that $m \ell \equiv 1 \bmod 2 n$. Then $\sum_{i=0}^{\ell-1}\left(-x^{m}\right)^{i}$ is the inverse of $u_{m} \bmod \left\langle\Sigma^{-}\right\rangle$.

Lemma 1.4 If $m \geq 1$ and $(m, 2 n)=1$, then the left ideal $\langle m, x+1\rangle$ of $\mathbb{Z} Q_{4 n}$ is generated by $u_{m}$ or $z_{m}$ and is isomorphic to $\mathbb{Z} Q_{4 n}$.

Proof Since $m \equiv u_{m} \bmod \langle x+1\rangle$ and $\Sigma^{-}$annihilates $x+1$, this ideal is generated by $u_{m}$.

For the purpose of constructing examples of interest in topology, as in [3] we now provide some explicit isomorphisms. Under easily verifiable hypotheses, these show that $P$ is projective and even stably free. Theorem 1.6 will show that the isomorphism class of $P$ has order at most 2 in $C\left(\mathbb{Z} Q_{4 n}\right)=\widetilde{K}_{0}\left(\mathbb{Z} Q_{4 n}\right)$.

Lemma 1.5 If $(k, 2 n)=1$, there are polynomials $A(x), B(x), C(x)$ and $D(x)$ that, considered as elements of $\mathbb{Z} Q_{4 n}$, satisfy the following:

(i) $A(x) \equiv a \bmod \left\langle(x+1)^{2}\right\rangle$ and $B(x) \equiv b \bmod \left\langle(x+1)^{2}\right\rangle$;

(ii) both $A(x)$ and $B(x)$ commute with $y$;

(iii) $\quad D(x)=[A(x)]^{2}-[B(x)]^{2} x^{n}-C(x)(x+1)^{2}$ is a unit in $\mathbb{Z} Q_{4 n} /\left\langle\Sigma^{-}\right\rangle$, where

$$
D(x):=\operatorname{sgn}(k)\left(1+\sum_{m=1}^{\frac{|k|-1}{2}}(-1)^{m}\left[x^{-m}+x^{m}\right]\right)=\operatorname{sgn}(k) z_{|k|} .
$$

Proof First note that, in $\mathbb{Z}\left[x, x^{-1}\right], x^{-m}+2+x^{m} \equiv 0 \bmod \left\langle(x+1)^{2}\right\rangle$ when $m$ is odd and $x^{-m}-2+x^{m} \equiv 0 \bmod \left\langle(x+1)^{2}\right\rangle$ when $m$ is even. Also note that $\left\langle(x+1)^{2}\right\rangle$, considered as a left ideal of $\mathbb{Z} Q_{4 n}$, is actually a two-sided ideal. In addition, $D(x)$ is a unit in $\mathbb{Z} Q_{4 n} /\left\langle\Sigma^{-}\right\rangle$by Lemma 1.3. 
Case 1: $\mathrm{n}$ odd Then $k=a^{2}+b^{2}$. Since $y(a+b y)=a y+b x^{n} \equiv-b+a y$ $\bmod \langle x+1\rangle$, it suffices to consider $a$ odd. Then working $\bmod \left\langle(x+1)^{2}\right\rangle$ in $\mathbb{Z} Q_{4 n}$,

$$
\begin{aligned}
& 0 \equiv \sum_{m=1}^{\frac{a^{2}-1}{2}}(-1)^{m}\left[x^{-m}+(-1)^{m+1} 2+x^{m}\right] \\
&+\sum_{m=\frac{a^{2}+1}{2}}^{\frac{k-1}{2}}(-1)^{m}\left[x^{n-m}+(-1)^{m-n+1} 2+x^{m-n}\right] x^{n} \\
& \equiv-1+\left(1+\sum_{m=1}^{\frac{k-1}{2}}(-1)^{m}\left[x^{-m}+x^{m}\right]\right)-2 \frac{a^{2}-1}{2}+2 \frac{b^{2}}{2} x^{n} \\
&=D(x)-a^{2}+b^{2} x^{n} .
\end{aligned}
$$

Case 2: $\mathrm{n}$ even Then $k=a^{2}-b^{2}$. Since $y(a+b y)=a y+b x^{n} \equiv b+a y \bmod \langle x+1\rangle$, without loss of generality, $b$ is even. Again, working $\bmod \left\langle(x+1)^{2}\right\rangle, 2\left(1-x^{n}\right)=$ $\left(1-x^{n}\right)^{2} \equiv 0$ and

$$
\begin{aligned}
0 & \equiv \sum_{m=1}^{\frac{|k|-1}{2}}(-1)^{m}\left[x^{-m}+(-1)^{m+1} 2+x^{m}\right] \mp \frac{b^{2}}{2}\left(1-x^{n}\right)^{2} \\
& =-1+\left(1+\sum_{m=1}^{\frac{|k|-1}{2}}(-1)^{m}\left[x^{-m}+x^{m}\right]\right)-2 \frac{\left|a^{2}-b^{2}\right|-1}{2} \mp b^{2} \pm b^{2} x^{n} \\
& = \pm\left[D(x)-a^{2}+b^{2} x^{n}\right] .
\end{aligned}
$$

Use the upper signs if $k>0$, the lower signs if $k<0$.

Let $A(x)=a$ and $B(x)=b$. The previous computations show that is possible to find an appropriate $C(x)$. In practice, other choices for $A(x)$ and $B(x)$ may allow a shorter expression for $C(x)$ as in Example 2.4.

Theorem 1.6 If $(k, 2 n)=1$ and $A(x), B(x), C(x), D(x)$ are polynomials satisfying the conditions of Lemma 1.5, then the map $\phi: \mathbb{Z} Q_{4 n} \oplus \mathbb{Z} Q_{4 n} \rightarrow \mathbb{Z} Q_{4 n} \oplus \mathbb{Z} Q_{4 n}$ given by right multiplication with the matrix

$$
\Phi(a, b, n)=\left[\begin{array}{cc}
A(x)+B(x) y & C(x)(x+1) \\
x+1 & A(x)-B(x) x y
\end{array}\right]
$$

gives an isomorphism $\mathbb{Z} Q_{4 n} \oplus \mathbb{Z} Q_{4 n} \cong P \oplus P$. Thus $P$ has order at most 2 in the reduced class group. In addition, $\Phi$ is invertible $\bmod \left\langle\Sigma^{-}\right\rangle$. 
Proof The following argument relies on repeated use of the properties (i)-(iii) of Lemma 1.5. Note that $\operatorname{im} \phi \subseteq P \oplus P$. Since $[-(x+1), A(x)+B(x) x y] \Phi=[0, D(x)]$, it follows that $[0, x+1] \in \operatorname{im} \phi$. Similarly, $[A(x)-B(x) y,-C(x)(x+1)] \Phi \equiv[D(x), 0]$ mod $[0, x+1]$ implies $[D(x), 0]$ and $[x+1,0]$ lie in im $\phi$. Consequently $[a+b y, 0]$ and $[0, a+b y]$ are contained in the image. Thus $\operatorname{im} \phi=P \oplus P$. Since $P$ and $\mathbb{Z} Q_{4 n}$ have the same rank as abelian groups, $\phi$ is a monomorphism.

Since $[D(x), 0]$ and $[0, D(x)]$ lie in $\operatorname{im} \phi, \Phi$ has a left inverse $\bmod \left\langle\Sigma^{-}\right\rangle$. By Remark 1.1 and Lemma 1.2, $\Phi$ is invertible $\bmod \left\langle\Sigma^{-}\right\rangle$.

Corollary 1.7 If $(k, 2 n)=1$ and $a$ is odd, a particular isomorphism $\Phi(a, b, n)$ in Theorem 1.6 is given by the matrix

$$
\Phi(a, b, n)=\left[\begin{array}{cc}
a+b y & C(x)(x+1) \\
x+1 & a-b x y
\end{array}\right]
$$

where $C(x):=-(x+1)^{-2}\left\{\sum_{m=1}^{\frac{a^{2}-1}{2}}(-1)^{m}\left[x^{-m}+(-1)^{m+1} 2+x^{m}\right]\right.$

$$
\left.+\sum_{m=\frac{a^{2}+1}{2}}^{\frac{k-1}{2}}(-1)^{m}\left[x^{n-m}+(-1)^{m-n+1} 2+x^{m-n}\right] x^{n}\right\}
$$

when $n$ is odd, and

$C(x):=-\operatorname{sgn}(k)(x+1)^{-2}\left\{\sum_{m=1}^{\frac{|k|-1}{2}}(-1)^{m}\left[x^{-m}+(-1)^{m+1} 2+x^{m}\right] \mp \frac{b^{2}}{2}\left(1-x^{n}\right)^{2}\right\}$

when $n$ is even. In both cases $C(x)$ is an integral Laurent polynomial in $x$. When $C(x)$ is interpreted as an element of $\mathbb{Z} Q_{4 n}$,

$$
C(x)(x+1)^{2}=a^{2}-b^{2} x^{n}-\operatorname{sgn}(k) z_{|k|} .
$$

Proof Note that $b$ is even and that each individual summand within the braces of the definition of $C(x)$ is divisible by $(x+1)^{2}$ in $\mathbb{Z}\left[x, x^{-1}\right]$.

\section{A class of stably free $\mathbb{Z} Q_{4 n}$-modules: constructing isomor- phisms}

This section gives a free basis for a stabilization of $P$, which will be used in Section 4 to define the second boundary map of an exotic algebraic 2-complex. 
We continue using the notation of Section 1 and only consider

$$
P=P_{a+b y}=\langle a+b y, x+1\rangle
$$

under the condition $(k, 2 n)=1$. When $a+b y$ is of the form $h(e+f y)^{2}$ in $\left(\mathbb{Z}_{n}[y] /\left\langle y^{2}-(-1)^{n}\right\rangle\right)^{*}$ for some integers $e, f$, and $h$, it is possible to directly construct an isomorphism $\mathbb{Z} Q_{4 n} \oplus \mathbb{Z} Q_{4 n} \cong \mathbb{Z} Q_{4 n} \oplus P$. This gives an elementary proof that $P$ is stably free. This construction is motivated by attempting to lift the isomorphism in $G L_{2}\left(\mathbb{Z}_{n}[y] /\left\langle y^{2}-(-1)^{n}\right\rangle\right)$ given by

$$
\left[\begin{array}{cc}
1 & 0 \\
0 & a+b y
\end{array}\right]=\left[\begin{array}{cc}
(e+f y)^{-1} & 0 \\
0 & e+f y
\end{array}\right]\left[\begin{array}{cc}
e+f y & 0 \\
0 & e+f y
\end{array}\right]\left[\begin{array}{ll}
1 & 0 \\
0 & h
\end{array}\right] .
$$

Similar to the definition of $k=a^{2} \pm b^{2}$, let $\bar{k}=e^{2} \pm f^{2}$, depending on whether $n$ is odd or even. Then $k=h^{2} \bar{k}^{2}$. For easier computation, define for integers $j$ :

$$
X(j)=\left\{\begin{array}{cc}
\operatorname{sgn}(j) \sum_{\ell=0}^{|j|-1}(-x)^{\ell n}, & \text { if } j \neq 0 ; \\
0, & \text { if } j=0 .
\end{array}\right.
$$

Then the following holds:

$$
\begin{gathered}
X(1)=1 \text { and } X(-j)=-X(j) \text { for all } j, \\
x^{n} X(j)=(-1)^{n} X(j) \text { for } j \text { even, } \\
X(i) X(j)=X(i \cdot j) \text { for all } i \text { and } j \\
X(i)+X(j)=X(i+j) \text { if at least one of } i \text { or } j \text { is even, } \\
d \cdot X(j)=X(d \cdot j) \text { for } j \text { even and } d \text { an integer. }
\end{gathered}
$$

Also note that $X(j) \equiv j \bmod \langle x+1\rangle$ and that $X(j)$ lies in the commutative subring generated by $y$.

Lemma 2.1 Recall $\bar{k}=e^{2} \pm f^{2}$ and $k=h^{2} \bar{k}^{2}$. Let $(k, 2 n)=1$ and $c$ be an integer such that $c \bar{k} \equiv 1 \bmod 4 n^{2}$ and $c \bar{k}>0$. There is an matrix $\Psi$ over $\mathbb{Z} Q_{4 n}$ with the following properties:

(a) $\Psi$ is invertible $\bmod \left\langle\Sigma^{-}\right\rangle$.

(b) $\Psi \equiv\left[\begin{array}{cc}c(e-f y) & 0 \\ 0 & e+f y\end{array}\right] \bmod \langle x+1\rangle$.

In particular, with $d=\frac{c \bar{k}-1}{4 n^{2}} \geq 0$, the following matrix has these properties:

$$
\Psi(e, f, n)=\left[\begin{array}{cc}
X(c)(X(e)-X(f) y) & X(2 n)-\Sigma^{-} \\
d\left(X(2 n)-\Sigma^{-}\right) & X(e)+X(f) y
\end{array}\right] .
$$

Algebraic $\mathcal{B}$ Geometric Topology, Volume 8 (2008) 
Proof The entries of $\Psi(e, f, n)$ lie in the commutative subring generated by $y$ and $\Sigma^{-}$. Using (2-2) to (2-6), one may show that the determinant $\bmod \left\langle\Sigma^{-}\right\rangle$is \pm 1 or $\pm x^{n}$.

Note that Lemma 1.3 implies that

$$
\Delta(h)=\left[\begin{array}{cc}
1 & 0 \\
0 & z_{h}
\end{array}\right]
$$

is invertible $\bmod \left\langle\Sigma^{-}\right\rangle$whenever $(h, 2 n)=1$, while $\Delta(1)$ is the identity matrix.

Theorem 2.2 Let $(k, 2 n)=1$ and $a+b y=h(e+f y)^{2}$ in $\mathbb{Z}_{n}[y] /\left\langle y^{2}-(-1)^{n}\right\rangle$ for integers $e, f, h$. Then $P=\langle a+b y, x+1\rangle$ is stably free. In particular, with $c$ and $d$ as in Lemma 2.1, let $\Psi(e, f, n), \Phi(e, f, n), \Delta(h)$ be as in (2-7), (1-2), (2-8), respectively. Let $v=1-2 d n \Sigma^{-}+4 d n^{2}$. Then the product matrix

$$
\Theta=\Psi(e, f, n) \Phi(e, f, n) \Delta(h)\left[\begin{array}{cc}
\frac{v}{1+4 d n^{2}} & 0 \\
0 & 1
\end{array}\right]
$$

has entries in $\mathbb{Z} Q_{4 n}$. The map $\theta: \mathbb{Z} Q_{4 n} \oplus \mathbb{Z} Q_{4 n} \rightarrow \mathbb{Z} Q_{4 n} \oplus \mathbb{Z} Q_{4 n}$ defined by multiplying row vectors on the right by $\Theta$ is a monomorphism with image $\mathbb{Z} Q_{4 n} \oplus P$.

Proof The map $\bar{\theta}: \mathbb{Z} Q_{4 n} \oplus \mathbb{Z} Q_{4 n} \rightarrow \mathbb{Z} Q_{4 n} \oplus \mathbb{Z} Q_{4 n}$, defined by multiplying row vectors on the right by $\Psi(e, f, n) \Phi(e, f, n) \Delta(h)$, is invertible mod $\Sigma^{-}$. Hence the vectors $[x+1,0]$ and $[0, x+1]$ are contained in the image. Thus $[c(e+f y)(e-f y), 0]$ and $\left[0, h(e+f y)^{2}\right]$ are contained in $\operatorname{im} \bar{\theta} \bmod \langle x+1\rangle$. As in the proof of Theorem 1.6, it follows that the image of $\bar{\theta}$ is $\langle m, x+1\rangle \oplus P$ with $m=c\left[e^{2}+(-1)^{n+1} f^{2}\right]=c \bar{k}>0$. The restriction of $\bar{\theta}$ in the codomain is an isomorphism $\mathbb{Z} Q_{4 n} \oplus \mathbb{Z} Q_{4 n} \cong\langle m, x+1\rangle \oplus P$. By Lemma $1.4\langle m, x+1\rangle$ is generated by

$$
u_{m}=\sum_{i=0}^{m-1}(-1)^{i} x^{i}=1+2 d n \Sigma^{-},
$$

and therefore free. Thus $P$ is stably free. Then $u_{m} v=1+4 d n^{2}$, and $\bar{\theta}$ can be adjusted to $\theta$ so that $\operatorname{im} \theta$ is exactly equal to $\mathbb{Z} Q_{4 n} \oplus P \subseteq \mathbb{Z} Q_{4 n} \oplus \mathbb{Z} Q_{4 n}$ by redefining its matrix as $\Theta$. Computations in the rational group ring show that the entries of $\Theta$ are actually in $\mathbb{Z} Q_{4 n}$.

Corollary 2.3 The matrix $\Theta$ of Theorem 2.2 is invertible in $\mathbb{Z} Q_{4 n} /\left\langle\Sigma^{-}\right\rangle$.

Proof Note that $1+2 d n \Sigma^{-}$is the inverse of $\frac{v}{1+4 d n^{2}}$ in the rational group ring. 
Example 2.4 Let $n \geq 3$ be odd with $(n, 5)=1$. Let $P=\langle-3+4 y, x+1\rangle \subseteq \mathbb{Z} Q_{4 n}$, where $-3+4 y=(1+2 y)^{2}$ in $\mathbb{Z}_{n}[y] /\left\langle y^{2}+1\right\rangle$. Let $m=\frac{n-1}{2}$ and $\ell=n-2$. For $n^{2} \equiv \pm 1 \bmod 5$, set

$$
B=2\left(n^{2} \mp 1\right) / 5, \quad A=B \pm 1, \quad \text { and } \quad w=\left( \pm 1-2 n \Sigma^{-}+4 n^{2}\right) /\left(1 \pm 4 n^{2}\right) .
$$

With these choices of $A(x)=A$ and $B(x)=B$, the monomorphism $\theta$ of Theorem 2.2 is given by multiplying row vectors on the right by

$$
\begin{aligned}
\Theta=\left[\begin{array}{cc}
1-\left(1+x^{n}\right) y & n-n x^{n}-\Sigma^{-} \\
n-n x^{n}-\Sigma^{-} & \left(A-B x^{n}\right)\left(1-\left(1-x^{n}\right) y\right)
\end{array}\right] \times & \\
& {\left[\begin{array}{cc}
-x^{n}\left(1+x^{-\ell}\right) & 1+(-1)^{m}\left(x^{-m}+x^{m}\right) y \\
1-(-1)^{m}\left(x^{-m}+x^{m}\right) x^{\ell} y & \left(x^{\ell}+1\right)
\end{array}\right]\left[\begin{array}{ll}
w & 0 \\
0 & 1
\end{array}\right] . }
\end{aligned}
$$

Example 2.5 Let $n=p$ be an odd prime. When $p \equiv 3 \bmod 4$, all elements of $\mathbb{F}_{p}^{*}$ are squares in $\left(\mathbb{F}_{p}[y] /\left\langle y^{2}+1\right\rangle\right)^{*} \cong \mathbb{F}_{p^{2}}^{*}$. When $p \equiv 1 \bmod 4,\left(\mathbb{F}_{p}[y] /\left\langle y^{2}+1\right\rangle\right)^{*} \cong$ $\mathbb{F}_{p}^{*} \times \mathbb{F}_{p}^{*}$, where $1 \mapsto(1,1)$ and $y \mapsto(\alpha,-\alpha)$ with $\alpha^{2}=-1$. It is possible to have $P_{a+b y}$ stably free when $a+b y$ is not a square. For $p=13$, choose $\alpha=5$. Then $\left(\mathbb{F}_{13}[y] /\left\langle y^{2}+1\right\rangle\right)^{*}$ is generated by $-5+4 y$ and $-5-4 y$ corresponding to $(2,1)$ and $(1,2)$ in $\mathbb{F}_{13}^{*} \times \mathbb{F}_{13}^{*}$, respectively. The element $5+2 y=(-5+4 y)(-5-4 y)^{3}$ corresponding to $(2,8)$ is not a square, but $P_{5+2 y}$ is stably free by Theorem 2.2 with $h=41$.

\section{Stably free modules over $\mathbb{Z} Q_{4 n}$ that are not free}

When $P \oplus \mathbb{Z} Q_{4 n} \cong \mathbb{Z} Q_{4 n} \oplus \mathbb{Z} Q_{4 n}$, then there is an algebraic 2-complex whose second homology module is isomorphic to $P / N P$. This is part of our argument [3,p 901f] which is based on Swan [17, Lemma 2.1] and the fact that $Q_{4 n}$ is the fundamental group of a compact 3-manifold. This section gives criteria for determining whether or not $P$ is free. To this end, we give conditions for $P / N P$ not to be singly generated as a $\mathbb{Z} Q_{4 n}$-module, which will imply that $P / N P$ cannot serve as the second homotopy group of a known geometric 2-complex. These results are not exhaustive. Rather, the intent is to give easily testable criteria which often answer this question and provide examples of stably free nonfree modules over $Q_{4 n}$ for infinitely many $n$. This collection of examples is large enough to include groups where $K_{1}\left(\mathbb{Z} Q_{4 n}\right)$ is not represented by units. We extend the results of Swan [20, Chapter 10] by not requiring certain technical assumptions on the real units of cyclotomic integers. 
Example 3.1 Swan [20, Theorem I, p 66] has shown that all stably free modules are free for $Q_{8}, Q_{12}, Q_{16}$, and $Q_{20}$. Thus for $n \in\{2,3,4,5\}, P$ is free whenever $P$ is stably free.

Lemma 3.2 Let $(k, 2 n)=1$. Then $P=\langle a+b y, x+1\rangle$ is singly generated if, and only if, there exists a $\mu \in \mathbb{Z} Q_{4 n}$ such that $\mu \bmod \left\langle\Sigma^{-}\right\rangle$is a unit in $\mathbb{Z} Q_{4 n} /\left\langle\Sigma^{-}\right\rangle$and $\mu \equiv a+b y \bmod \langle x+1\rangle$.

Proof Note that $\left\langle\Sigma^{-}\right\rangle$is the annihilator of $\langle x+1\rangle$. The remainder of this proof is very similar to that of the following lemma and is omitted here.

Lemma 3.3 Let $(k, 2 n)=1, \Xi=(1+y) \sum_{\ell=0}^{n-1} x^{2 \ell}$, and $P=\langle a+b y, x+1\rangle$. Then $P / N P$ is singly generated if, and only if, there exists a $\mu \in \mathbb{Z} Q_{4 n}$ such that $\mu$ represents a unit in $\mathbb{Z} Q_{4 n} /\langle\Xi\rangle$ and $\mu \equiv a+b y \bmod \langle x+1\rangle$.

Proof Recall that $\langle\Xi\rangle$ is a two-sided ideal of $\mathbb{Z} Q_{4 n}$, and note that $\langle\Xi\rangle /\langle N\rangle$ is the (two-sided) annihilator of $\langle x+1\rangle /\langle N\rangle$ in $\mathbb{Z} Q_{4 n} /\langle N\rangle$.

If such a $\mu$ exists, then $\mu \in P$ and $P=\langle\mu, x+1\rangle$, and there is a $\gamma \in \mathbb{Z} Q_{4 n}$ such that $\gamma \mu=1 \bmod \langle\Xi\rangle$. Thus $(x+1) \gamma \mu \equiv x+1 \bmod \langle N\rangle$ and $\mu$ generates $P / N P$.

Conversely, suppose $\eta \bmod \langle N\rangle$ generates $P / N P$, where $\eta=\sigma(a+b y)+\rho(x+1)$ with $\sigma, \rho \in \mathbb{Z} Q_{4 n}$. Since $P$ and $\langle x+1\rangle$ are two-sided ideals, $\eta$ can be rewritten as $(a+b y)(c+d y)+\rho^{\prime}(x+1)$ with $c, d$ integers and $\rho^{\prime} \in \mathbb{Z} Q_{4 n}$. Since $P / N P=$ $\langle\eta\rangle,\langle a+b y\rangle \bmod \langle x+1\rangle=\langle\eta\rangle \bmod \langle x+1\rangle$ in $\mathbb{Z} Q_{4 n} /\langle x+1\rangle$. When $n$ is odd, there are no zero divisors in $\mathbb{Z} Q_{4 n} /\langle x+1\rangle$, the Gaussian integers. When $n$ is even, $\mathbb{Z} Q_{4 n} /\langle x+1\rangle \cong \mathbb{Z} C_{2}$ and $a+b y \bmod \langle x+1\rangle$ is not a zero divisor because $k=a^{2}-b^{2} \neq 0$ by assumption. In either case, $\mathbb{Z} Q_{4 n} /\langle x+1\rangle$ has only trivial units $\{ \pm 1, \pm y\}$. Hence $P / N P$ will always have a generator represented by $\eta^{\prime}=(a+b y)+\rho^{\prime \prime}(x+1)$, where $\rho^{\prime \prime} \in \mathbb{Z} Q_{4 n}$. Thus $\gamma \eta^{\prime} \equiv\langle x+1\rangle \bmod \langle N\rangle$, for some $\gamma \in \mathbb{Z} Q_{4 n}$ and $\gamma \eta^{\prime} \equiv \gamma(a+b y) \equiv 0 \bmod \langle x+1\rangle$. Therefore $\gamma=(x+1) \gamma^{\prime}$ for some $\gamma^{\prime} \in \mathbb{Z} Q_{4 n}$. Then $\gamma^{\prime} \eta^{\prime} \equiv 1 \bmod \langle\Xi\rangle$. Then $\gamma^{\prime}$ is the inverse of $\eta^{\prime}$ in $\mathbb{Z} Q_{4 n} /\left\langle\Sigma^{-}\right\rangle$by Remark 1.1 and Lemma 1.2.

Theorem 3.4 Let $(k, 2 n)=1$. If $2 n \mid a$ or $2 n \mid b$, then $P$ is free.

Proof Suppose $2 n \mid a$. With the notation of Section 1,

$$
P=\left\langle u_{a}+u_{b} y, x+1\right\rangle=\left\langle\frac{a}{2 n} \Sigma^{-}+u_{b} y, x+1\right\rangle=\left\langle\frac{a}{2 n} \Sigma^{-}+u_{b} y\right\rangle
$$

since $(b, 2 n)=1$ implies $u_{b}$ is a unit $\bmod \left\langle\Sigma^{-}\right\rangle$. Thus $P$ is singly generated, hence free. The case $2 n \mid b$ is similar. 
For the remainder of this paper, we restrict attention to odd $n$. Studying the image of $\mathbb{Z} Q_{4 n} /\left\langle\Sigma^{-}\right\rangle$in appropriate quotient rings, we will give conditions under which $P$ is not free. To this end, let $m>1$ be a divisor of $n$. Then there is a homomorphism of $\mathbb{Z} Q_{4 n}$ onto $\mathbb{Z} Q_{4 m}$ which induces a homomorphism of $\mathbb{Z} Q_{4 n} /\left\langle\Sigma^{-}\right\rangle$onto $\Lambda_{2 m}=\mathbb{Z} Q_{4 m} /\left\langle\Phi_{2 m}(x)\right\rangle \cong \mathbb{Z} Q_{4 n} /\left\langle\Phi_{2 m}(x)\right\rangle$, where $\Phi_{2 m}(x)$ is the $2 m$ th cyclotomic polynomial and $\Phi_{1}(x)=x-1$. Moreover, $\Lambda_{2 m}$ is a $\mathbb{Z}$-order in the totally definite quaternion algebra $\mathbb{H}_{2 m}=\mathbb{Q} \otimes \Lambda_{2 m}$ by Swan [20, Lemma 4.2]. Let $\zeta_{2 m}$ be a primitive $2 m$ th root of unity. $\Lambda_{2 m}$ may be identified with $\mathbb{Z}\left[\zeta_{2 m}\right]+\mathbb{Z}\left[\zeta_{2 m}\right] y$ where $y^{2}=-1$ and $y a=\bar{a} y$ for $a \in \mathbb{Z}\left[\zeta_{2 m}\right]$, cf [20, p 75]. Under this identification, the center of $\Lambda_{2 m}$ is $R_{2 m}=\mathbb{R} \cap \mathbb{Z}\left[\zeta_{2 m}\right]=\mathbb{Z}\left[\tau_{2 m}\right]$ where $\tau_{2 m}=\zeta_{2 m}+\zeta_{2 m}^{-1}$.

The proof of [20, Lemma 10.13] contains sufficient information for criteria that imply $P$ is not free provided a certain restrictive condition on the totally positive units of $R_{2 p}$ is met for some prime $p \mid n$, namely, $\left(R_{2 p}^{*}\right)^{2}=\left(R_{2 p}^{*}\right)^{+}$. Our proof is independent of this condition, leading to the easily testable criterion of Theorem 3.11.

Lemma 3.5 (Magurn-Oliver-Vaserstein [13, Lemma 7.5(b)]) Assume $m>1$ is odd. Then $\Lambda_{2 m}^{*}$ is generated by $\mathbb{Z}\left(\zeta_{2 m}\right)^{*}$ and $y$.

Corollary 3.6 When $m \mid n$ and $m>1$, every unit $u$ in $\mathbb{Z} Q_{4 n} /\left\langle\Phi_{2 m}(x)\right\rangle$ can be written in the form $D(x)$ or $D(x) y$ for a suitable polynomial $D(x)$, depending on $u$.

For the remainder of this section, the following assumption holds:

Assumption 3.7 $G$ is a group generated by $x$ and $y$, where $x$ has finite order $t>1$ and generates a subgroup $C_{t}$ of index 2 .

Consider an integral polynomial $p(x)$ also as an element of $\mathbb{Z} C_{t} \subset \mathbb{Z} G$ and let $\langle p(x)\rangle$ denote the left ideal in $\mathbb{Z} G$. Such a polynomial may satisfy the condition:

Assumption 3.7 holds; $p(x)$ is a divisor in $\mathbb{Z}[x]$ of $x^{t}-1$; deg $p(x)>0$;

(*) and every unit $u$ in $\mathbb{Z} G /\langle p(x)\rangle$ can be written in the form $D(x)$ or $D(x) y$ for a suitable polynomial $D(x)$, depending on $u$.

Note that Assumption 3.7 implies that $C_{t}$ is a normal subgroup of $G$ and thus $y x y^{-1}=$ $y^{-1} x y=x^{r}$ with $(r, t)=1$. The second clause of $(*)$ means that $p(x)$ is a product of distinct cyclotomic polynomials $\Phi_{s}(x)$ where $s \mid t$. Let $\zeta$ denote a primitive $s$ th root of unity. Then $\zeta^{r}$ is also a primitive $s$ th root of unity. Thus $\Phi_{s}\left(\zeta^{r}\right)=0$ and $\zeta$ is a root of $\Phi_{s}\left(x^{r}\right)$. Since $\Phi_{S}(x)$ is irreducible over $\mathbb{Q}, \Phi_{S}(x) \mid \Phi_{s}\left(x^{r}\right)$. Therefore $\left\langle\Phi_{s}(x)\right\rangle$ and also $\langle p(x)\rangle$ are two-sided ideals in $\mathbb{Z} G$.

Corollary 3.6 implies that $\Phi_{2 m}(x)$ satisfies (*) when $n$ is odd, $m>1$, and $m \mid n$ with $G=\mathbb{Z} Q_{4 n}$. 
Lemma 3.8 Let $G$ satisfy the above Assumption 3.7. Assume that the integral polynomials $p(x)$ and $q(x)$ satisfy $(*)$ and that the ideal $I=\langle p(x), q(x)\rangle$ in $\mathbb{Z}[x]$ is not $\mathbb{Z}[x]$, while its rationalization $\mathbb{Q} I$ equals $\mathbb{Q}[x]$. Then $p(x) q(x)$ also satisfies $(*)$.

Proof Let $\mu \in \mathbb{Z} G$, and suppose that $\mu=A(x)+B(x) y$ represents a unit in $\mathbb{Z} G /\langle p(x) q(x)\rangle$ for some polynomials $A(x), B(x)$ in $\mathbb{Z}[x]$. Since $\mu$ must map to a unit in $\mathbb{Z} G /\langle p(x)\rangle$, either $A(x) \equiv 0 \bmod \langle p(x)\rangle$ or $B(x) \equiv 0 \bmod \langle p(x)\rangle$. Similarly for $\mathbb{Z} G /\langle q(x)\rangle$. Thus if $\mu$ has the form $c(x) p(x)+d(x) q(x) y$ or $c(x) q(x)+$ $d(x) p(x) y$ for some $c(x), d(x)$ then $\langle p(x), q(x)\rangle=\mathbb{Z} G$. There remains the case that $A(x)=\alpha(x) p(x)=\beta(x) q(x)$, where $\alpha(x), \beta(x)$ are in $\mathbb{Z}[x]$ (or the analogous case for $B(x))$. Since $\mathbb{Q} I=\mathbb{Q}[x]$, there exist $u(x), v(x)$ in $\mathbb{Z}[x]$ such that

$$
u(x) p(x)+v(x) q(x)=j \in \mathbb{Z} \backslash\{0\}
$$

and $q(x) \mid j \alpha(x)$. Because $q(x)$ is an integral polynomial with leading coefficient \pm 1 , the Euclidean algorithm gives $\alpha(x)=s(x) q(x)+r(x)$, where $s(x), r(x)$ are integral polynomials with $r(x)=0$ or $\operatorname{deg} r(x)<\operatorname{deg} q(x)$. It follows that $\langle p(x)\rangle \cap\langle q(x)\rangle=$ $\langle p(x) q(x)\rangle$ and either $A(x)$ or $B(x)$ must be a multiple of $p(x) q(x)$.

Theorem 3.9 In $\mathbb{Z}[x]$ the ideal $\left\langle\Phi_{m}(x), \Phi_{n}(x)\right\rangle$ is all of $\mathbb{Z}[x]$ unless $n=m p^{r}$ or $m=$ $n p^{r}$ for some prime $p$. If $m=n p^{r}$ with $r \geq 1$, then $\left\langle\Phi_{m}(x), \Phi_{n}(x)\right\rangle=\left\langle\Phi_{n}(x), p\right\rangle$.

Proof This is due to Diederichsen [5]; cf Swan [20, Appendix B, Theorem B1].

Corollary 3.10 Let $m>1$ be an integer and $L$ a finite set of integers $\ell>1$ such that either $\left\langle\Phi_{\ell}(x), \Phi_{m}(x)\right\rangle=\langle 1\rangle$ or $\ell / m$ is a proper power of some prime $p_{\ell}$. Let

$$
p(x)=\prod_{\ell \in L} \Phi_{\ell}(x) .
$$

Then

$$
\left\langle\Phi_{m}(x), p(x)\right\rangle=\left\langle\Phi_{m}(x), \prod p_{\ell}\right\rangle
$$

in $\mathbb{Z}[x]$. The latter product is indexed by all $\ell \in L$ for which $p_{\ell}$ is defined. (By convention, the product over the empty set denotes 1.)

Proof By induction on the number of elements of $L$. Note that if $p(x)=\Phi_{\ell}(x) q(x)$, then $\left\langle\Phi_{m}(x), p(x)\right\rangle=\left\langle\Phi_{m}(x),\left\langle\Phi_{m}(x), \Phi_{\ell}(x)\right\rangle q(x)\right\rangle$.

Theorem 3.11 Let $n>1$ be odd. Let $P$ be the left ideal $\langle a+b y, x+1\rangle \subseteq \mathbb{Z} Q_{4 n}$ and $\left(a^{2}+b^{2}, 2 n\right)=1$. If neither $a$ nor $b$ is a multiple of $n$, then $P$ is not free. In addition, $P / N P$ is not singly generated as a $\mathbb{Z} Q_{4 n}$-module. 
Proof Assume $P / N P$ is singly generated. By Lemma 3.3, there exists a $\mu \in \mathbb{Z} Q_{4 n}$ such that $\mu \bmod \langle\Xi\rangle$ is a unit in $\mathbb{Z} Q_{4 n} /\langle\Xi\rangle$ and $\mu \equiv a+b y \bmod \langle x+1\rangle$. Since

$\Xi=(1+y) \sum_{\ell=0}^{n-1} x^{2 \ell}=(1+y) \sum_{\ell=0}^{n-1} x^{\ell}\left(1-x+x^{2}-\cdots+x^{n-1}\right)=(1+y) \sum_{\ell=0}^{n-1} x^{\ell} \psi_{2 n}(x)$,

any such unit must map to a unit $\bar{\mu} \in \mathbb{Z} Q_{4 n} /\left\langle\psi_{2 n}(x)\right\rangle$ where $\mu \bmod \langle x+1\rangle=$ $a+b y \in\left(\mathbb{Z}_{n}[y] /\left\langle y^{2}+1\right\rangle\right)^{*}$. Here

$$
\psi_{2 n}(x)=\prod_{d \mid n, d \neq 1} \Phi_{2 d}(x) .
$$

Order the divisors of $n$ by increasing magnitude. For every proper divisor $r$ of $n$, there exists a prime $p$ such that $d=p r$ is also a divisor of $n$. First consider $\mathbb{Z} Q_{4 n} /\left\langle\Phi_{2 n}(x)\right\rangle$. If $\mu=A(x)+B(x) y$ is a unit in $\mathbb{Z} Q_{4 n} /\left\langle\Phi_{2 n}(x)\right\rangle$ for some polynomials $A(x), B(x)$, then by Corollary 3.6 either $A(x)$ or $B(x)$ is a multiple of $\Phi_{2 n}(x)$. For induction, observe: If

$$
q(x)=\prod_{d \mid n, r<d \leq n} \Phi_{2 d}(x)
$$

satisfies $(*)$ and $p(x)=\Phi_{2 r}(x)$ for $1<r<n$, then $p(x) q(x)$ satisfies ( $*$ ) by Lemma 3.8. Consequently, a finite number of induction steps gives that either $A(x)$ or $B(x)$ is a multiple of $\psi_{2 n}(x)$ and thus either $a=A(-1)$ or $b=B(-1)$ is a multiple of $n=\psi_{2 n}(-1)$, a contradiction. Thus $P / N P$ and the more $P$ are not singly generated as $\mathbb{Z} Q_{4 n}$-modules, so $P$ is not free as a $\mathbb{Z} Q_{4 n}$-module.

\section{Examples}

Example 4.1 Let $n \geq 7$ be an odd integer not divisible by 5. Let $P=\langle-3+4 y, x+1\rangle$ $\subseteq \mathbb{Z} Q_{4 n}$. Since $-3+4 y=(1+2 y)^{2} \in \mathbb{Z}_{n}[y] /\left\langle y^{2}+1\right\rangle$ and $(-3)^{2}+4^{2}=25, P$ is stably free by Theorem 2.2. By Theorem $3.11 P$ is not free and $P / N P$ is not singly generated.

Example 4.2 Let $n \geq 7$ be an odd integer divisible by 5 but not by 13 . Since $5+12 y=(3+2 y)^{2} \in \mathbb{Z}_{n}[y] /\left\langle y^{2}+1\right\rangle$ and $5^{2}+12^{2}=13^{2}, P=\langle 5+12 y, x+1\rangle \subseteq$ $\mathbb{Z} Q_{4 n}$ is stably free by Theorem 2.2. By Theorem 3.11P is not free and $P / N P$ is not singly generated.

Example 4.3 Let $n=5^{s} \ell$ be an odd natural number, where $s \geq 1,13 \mid \ell$ and $(\ell, 5)=1$. Use the Chinese Remainder Theorem to determine $e$ and $f \bmod 2 n$ where $e \equiv 3$ 
$\bmod 5^{s}, e \equiv 1 \bmod \ell, e \equiv 1 \bmod 2, f \equiv 2 \bmod 5^{s}, f \equiv 2 \bmod \ell, f \equiv 0 \bmod 2$. Let $a$ and $b$ be integers with $a \equiv e^{2}-f^{2} \bmod 2 n$ and $b \equiv 2 e f \bmod 2 n$. Then $a+b y=(e+f y)^{2} \in \mathbb{Z}_{n}[y] /\left\langle y^{2}+1\right\rangle$ and $P=\langle a+b y, x+1\rangle \subseteq \mathbb{Z} Q_{4 n}$ is stably free. By Theorem 3.11 $P$ is not free and $P / N P$ is not singly generated.

Theorem 4.4 (Swan [20, p 66]) If $n \geq 7$ is odd, there are stably free modules of rank 1 over $\mathbb{Z} Q_{4 n}$ which are not free.

Proof By Examples 4.1 to 4.3.

In the following, we use the alternative presentation

$$
Q_{4 n}=\left\langle x, y \mid x^{n} y^{-2}, y x y x^{-n+1}\right\rangle .
$$

Let $n \geq 3$ be an odd natural number. As in Theorem 2.2, let $a+b y=h(e+f y)^{2}$ with $\left(a^{2}+b^{2}, 1\right)=1$. Then $P_{a+b y}$ is stably free and the construction given in our previous paper [3] gives a free algebraic 2-complex over $Q_{4 n}$, ie, a truncated free resolution:

$$
\mathbb{Z} Q_{4 n}^{2} \stackrel{\partial_{2}}{\longrightarrow} \mathbb{Z} Q_{4 n}^{2} \stackrel{\partial_{1}}{\longrightarrow} \mathbb{Z} Q_{4 n} \stackrel{\varepsilon}{\longrightarrow} \mathbb{Z} \rightarrow 0,
$$

where $\varepsilon$ is augmentation and the boundary maps are given by multiplying row vectors on the right by matrices. The matrix for $\partial_{1}$ is $\left[\begin{array}{l}x-1 \\ y-1\end{array}\right]$ and the matrix for $\partial_{2}$ is

$$
\Theta\left[\begin{array}{cc}
\sum_{k=0}^{n-1} x^{k} & -(1+y) \\
y-\sum_{k=0}^{n-2} x^{k} & 1+y x
\end{array}\right]
$$

where $\Theta$ is given by (2-9).

Example 4.5 For $a+b y=-3+4 y$ and $n$ not a multiple of 5, Example 4.1 provides an infinite collection of such algebraic 2-complexes where $\Theta$ is explicitly given by the isomorphism of Example 2.4. When $P$ is given by Example 4.2 or Example 4.3, for the $\Theta$ of (2-9), choose $h=1$, the factor $\Phi(e, f, n)$ according to Corollary 1.7, the integer $\mathrm{c}$ and $\Psi(e, f, n)$ as in (2-7). In all of these cases $P / N P$ is not singly generated. Thus for all odd $n \geq 7$, this gives explicit algebraic 2-complexes for whose chain homotopy type the question of geometrization realization remains undecided. 


\section{References}

[1] P H Berridge, M J Dunwoody, Nonfree projective modules for torsion-free groups, J. London Math. Soc. (2) 19 (1979) 433-436 MR540056

[2] F R Beyl, M P Latiolais, N Waller, Classifications of 2-complexes whose finite fundamental group is that of a 3-manifold, Proc. Edinburgh Math. Soc. (2) 40 (1997) 69-84 MR1437812

[3] F R Beyl, N Waller, A stably free nonfree module and its relevance for homotopy classification, case $Q_{28}$, Algebr. Geom. Topol. 5 (2005) 899-910 MR2171797

[4] W J Browning, Homotopy types of certain finite $C W$-complexes with finite fundamental group, $\mathrm{PhD}$ thesis, Cornell University (1979)

[5] F-E Diederichsen, Über die Ausreduktion ganzzahliger Gruppendarstellungen bei arithmetischer Äquivalenz, Abh. Math. Sem. Hansischen Univ. 13 (1940) 357-412 MR0002133

[6] J Harlander, J A Jensen, Exotic relation modules and homotopy types for certain 1-relator groups, Algebr. Geom. Topol. 6 (2006) 2163-2173 MR2263062

[7] C Hog-Angeloni, W Metzler, A J Sieradski (editors), Two-dimensional homotopy and combinatorial group theory, London Mathematical Society Lecture Note Series 197, Cambridge University Press, Cambridge (1993) MR1279174

[8] F E A Johnson, Stable modules and the $\mathrm{D}(2)$-problem, London Mathematical Society Lecture Note Series 301, Cambridge University Press, Cambridge (2003) MR2012779

[9] F E A Johnson, Stable modules and Wall's D(2)-problem, Comment. Math. Helv. 78 (2003) 18-44 MR1966750

[10] F E A Johnson, Minimal 2-complexes and the D(2)-problem, Proc. Amer. Math. Soc. 132 (2004) 579-586 MR2022384

[11] I Kaplansky, Fields and rings, The University of Chicago Press, Chicago, Ill.-London (1969) MR0269449

[12] J Lewin, Projective modules over group-algebras of torsion-free groups, Michigan Math. J. 29 (1982) 59-64 MR646371

[13] B Magurn, R Oliver, L Vaserstein, Units in Whitehead groups of finite groups, J. Algebra 84 (1983) 324-360 MR723396

[14] MS Montgomery, Left and right inverses in group algebras, Bull. Amer. Math. Soc. 75 (1969) 539-540 MR0238967

[15] DS Passman, The algebraic structure of group rings, Pure and Applied Mathematics, Wiley-Interscience [John Wiley \& Sons], New York (1977) MR470211

[16] A J Sieradski, M N Dyer, Distinguishing arithmetic for certain stably isomorphic modules, J. Pure Appl. Algebra 15 (1979) 199-217 MR535186 
[17] R G Swan, Periodic resolutions for finite groups, Ann. of Math. (2) 72 (1960) 267-291 MR0124895

[18] R G Swan, Projective modules over group rings and maximal orders, Ann. of Math. (2) 76 (1962) 55-61 MR0139635

[19] R G Swan, $K$-theory of finite groups and orders, Lecture Notes in Mathematics 149, Springer, Berlin (1970) MR0308195 Notes by E Graham Evans

[20] R G Swan, Projective modules over binary polyhedral groups, J. Reine Angew. Math. 342 (1983) 66-172 MR703486

[21] M-F Vignéras, Simplification pour les ordres des corps de quaternions totalement définis, J. Reine Angew. Math. 286/287 (1976) 257-277 MR0429841

[22] C T C Wall, Finiteness conditions for CW-complexes, Ann. of Math. (2) 81 (1965) 56-69 MR0171284

[23] C T C Wall, Finiteness conditions for CW-complexes II, Proc. Roy. Soc. Ser. A 295 (1966) 129-139 MR0211402

Department of Mathematics and Statistics, Portland State University Portland, OR 97207-0751, USA

beylf@pdx.edu

Received: 5 July 2007 